\title{
Reassignment Scheme of an RFID Tag's Key for Owner Transfer
}

\author{
Junichiro Saito $^{1}$, Kenji Imamoto ${ }^{1}$, and Kouichi Sakurai ${ }^{2}$ \\ 1 Graduate School of Information Science and Electrical Engineering, \\ The Department of Computer Science and Communication Engineering, \\ Kyushu University, \\ 6-10-1 Hakozaki, Higashi-ku, Fukuoka, 812-8581, Japan \\ saito@itslab.csce.kyushu-u.ac.jp \\ imamoto@itslab.csce.kyushu-u.ac.jp \\ 2 Department of Computer Science and Communication Engineering, \\ Kyushu University, \\ 6-10-1 Hakozaki, Higashi-ku, Fukuoka, 812-8581, Japan \\ sakurai@csce.kyushu-u.ac.jp
}

\begin{abstract}
A Radio-Frequency-Identification (RFID) tag is a small and cheap device which is combined in IC chip and an antenna for radio communications. The RFID tag is used for management of goods and used as a substitute for a bar code. However, RFID system may infringe on a consumer's privacy because it has a strong tracing ability. In this paper, we propose a key change scheme which can prevent previous owner from reading the RFID tag after changing its owner. By using our scheme, previous owner cannot read and trace ID information on the RFID tag. Moreover it is possible to combine other privacy protection scheme with our scheme because our scheme uses only symmetric key cryptography.
\end{abstract}

\section{Introduction}

A Radio-Frequency-Identification (RFID) tag is a small and cheap device that consists of an IC chip and an antenna which communicate by radio frequency. A radio communication device called reader emits a query to an RFID tag and read ID of an RFID tag. When a reader emits a query, there are readers which also transmit power for the RFID tags, so an RFID tag does not have power supply in that case. Therefore an RFID tag expected to be used as a substitute of a bar code in the future. In order to use as a bar code, the cost of an RFID tag is $\$ 0.05 /$ unit, and tags are small as $0.4 \mathrm{~mm} * 0.4 \mathrm{~mm}$ and thin enough to be embedded in paper. For this reason, the capacity of a RFID tag is limited and an RFID tag is difficult to process complicated procedure. Moreover, because of using a radio frequency, a communication between an RFID tag and a reader will be simply monitored.

There are some kinds of an RFID tag and it is classified according to communication distance, the kind of memory, and the existence of a power supply. First, there are a close type $(0$ - several $\mathrm{mm})$ and proximity type (several $\mathrm{mm}$ - dozens $\mathrm{cm}$ ) and a remoteness type (dozens $\mathrm{cm}$ - several $\mathrm{m}$ ) in communication distance. In 
memory type, there are read only type, and once write and read type, and write and read type. In the case of writeable memory, a radio communication device called reader/writer can write in ID information on an RFID tag. In the case of read only type, the ID is determined in the manufacture stage of a RFID tag. Moreover, there are an active type and a passive type in the power supply of a RFID tag. The active type contains the power supply in the RFID tag, and a passive type obtains electric power from a reader by method which was mentioned above. From cost or the ease of use, a passive type of power supply is used in many cases.

This tag is used for next generation barcode in the field of distribution. By using an RFID tag, we can manage ID information in a database and optimize distribution and stock of products. An RFID tag has more information than printed barcode. Moreover, when an RFID tag is attached to various goods, consumers will use it after buying goods. For example, we can use an RFID tag for theft detection and goods management. More specificaly, a refrigerator which reads an RFID tag can observe best-before date of foodstuffs.

However, we should concern about security problem on an RFID tag. Most important problem is privacy problem. The communication between a reader and a RFID tag is performed by radio. Thus, it is simply tapped by an attacker. Moreover, the location of the owner can be traced by tracing the information on the specific RFID tag even if the attacker cannot understand the contents of ID. This privacy about owner's location is called as location privacy. For this reason, if an RFID tag is used for distribution, leakage of information about distribution is important. Moreover, a retailer can trace a consumer after selling goods. Therefore, we need a scheme to prevent from reading ID information on an RFID tag after changing its owner.

In this paper, we propose owner change schemes for an RFID tag by using three party model and two party model. In our schemes, ID information on an RFID tag is encrypted by using symmetric cryptosystem to prevent from leakage of ID information. Moreover, our schemes can prevent from reading ID information by previous owner by changing key of encryption.

\section{Privacy Problems on an RFID Tag}

The communication between a reader and an RFID tag is performed by radio. Thus, it is simply tapped by an attacker. The reader can simply derive information from the RFID tag and it can be used to infringement of the privacy. There are two privacy problems on the RFID tag. First is the leakage of ID information. Since the RFID tag has unique ID, if the attacker obtains the ID, he can get information about objects that the tag was attached. For example, the size and the price of clothes, the contents of a wallet, the inventory information about the goods of a store etc. can be leaked. As a result, it infringes on the owner's privacy. We can protect this problem by using anonymity of ID information by using encryption scheme. Therefore, the attacker can not know what encrypted ID means.

Second problem is the tracing ID information. An attacker can trace by tracing the information on the specific tag even if an attacker cannot understand the 


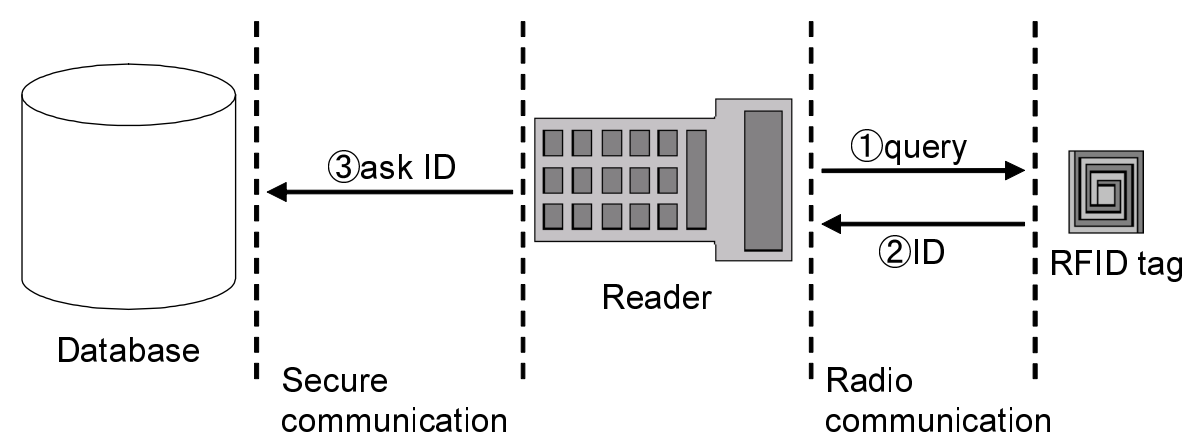

Fig. 1. RFID system

meanings of ID of the RFID tag. Therefore, he can know the location of the tag and the owner of the tag. This privacy about owner's location is called as location privacy. Therefore, an attacker can trace the owner of RFID tags by reading them. Moreover, it is also considered that the owner suffers the further damage. When a RFID tag is embedded in banknotes, it is possible that the information on an RFID tag can be read after drawing money from a bank, and it can be pursued exactly, and will be involved in a crime [1,2]. Even if the attacker can not know what kind of banknotes a person has, he can know the person has many tags which is attached to banknotes. So RFID tags can be a detector of rich people. Therefore, the effective privacy protection scheme in an RFID tag is needed. Against these problems, some previous results [3, 4, 5] proposed privacy protection schemes which can change ID information periodically to protect location privacy. Since ID information is not fixed by using these schemes, the attcker cannot trace specific tgas.

\subsection{Privacy Problems Related with Owner Changing}

When an RFID tag is used everywhere, the owner of the RFID tag is changed in its lifecycle. For example, when an RFID tag is attached to products, its owner changes from a manufacturer to a distributor, and from a retailer to a consumer. In this case, there are some problems if previous owner can read ID information on the RFID tag after changing its owner. For example, the retailer can trace the consumer. There are some schemes to prevent a third party from reading ID information [3, 4, 5]. However, since the previous owner might have important information like a decryption key, he can trace the RFID tag by using these information. Thus, we cannot protect consumer's privacy by using these schemes. Therefore, we need to change these information when owner of tags is changed.

\subsection{Traceability and Location Privacy}

We will show the diference between tracebility and location privacy. RFID tags realize traceability by tracing ID information on RFID tags. When RFID tags are 
used for goods management, reading ID information on RFID tags is recorded in a database and its source and transportation history are recorded. After goods are displayed at a shop, consumers can check a source of goods and know transportation history of the goods by accessing the database. In this case, this property that we can get backward information is called as traceability.

On the other hand, it is a problem that a shop assistant can trace RFID tags attached to goods by reading its ID information after consumers buy them. If the shop assistants can trace RFID tags, she can trace consumers and know the location of them. So we want that no one can get forward information after the owner of RFID tags is changed. So its privacy problem is called as location privacy.

Then, we propose schemes which can change a key on an RFID tag when its owner is changed. By using our schemes, previous owner cannot trace ID information on the RFID tag and we can protect privacy related with owner changing.

\section{Owner Change Scheme on Three Party Model}

We propose an owner change scheme using symmetric key cryptosystem. In our proposed scheme, an RFID tag is required to encrypt its ID information by using symmetric key. Moreover, our scheme can be used with other privacy protection scheme like 3, 4, 5] because we propose only key change scheme. So the ID information can be a ciphertext of these privacy protection scheme. In our scheme, previous owner gives an encryption key to new owner and new owner replaces the key to a new key. As a result, previous owner cannot decrypt ID information on the RFID tag by using his own key after changing the encryption key. Moreover, when new owner changes the key, we uses trusted third party (TTP) to encrypt new key. Therefore, new owner submits the encrypted key to the RFID tag. So if an attacker eavesdrops the encrypted key, she cannot decrypt the key. Therefore, we can prevent the attacker from getting new key.

Next, we define our model in our scheme and show our scheme.

\subsection{Model}

We show our model below. There are four entities in our model.

$-I D:$ ID is a static identifier, such that a serial number. Moreover, we can also use ciphertext of other privacy protection scheme as ID.

$-T$ : An RFID tag. It shares a symmetric key $K_{1}$ with Owner $_{1}$ and a symmetric key $K_{T T P}$ with $T T P$. It generates a ciphertext $C=S K_{K_{1}}[I D]$ by using $K_{1}$.

- Owner $_{1}$ : Previous owner. He shares a symmetric key $K_{1}$ with the RFID tag.

- Owner $_{2}$ : New owner. He receives $K_{1}$ from previous owner and generates new symmetric key $K_{2}$. 


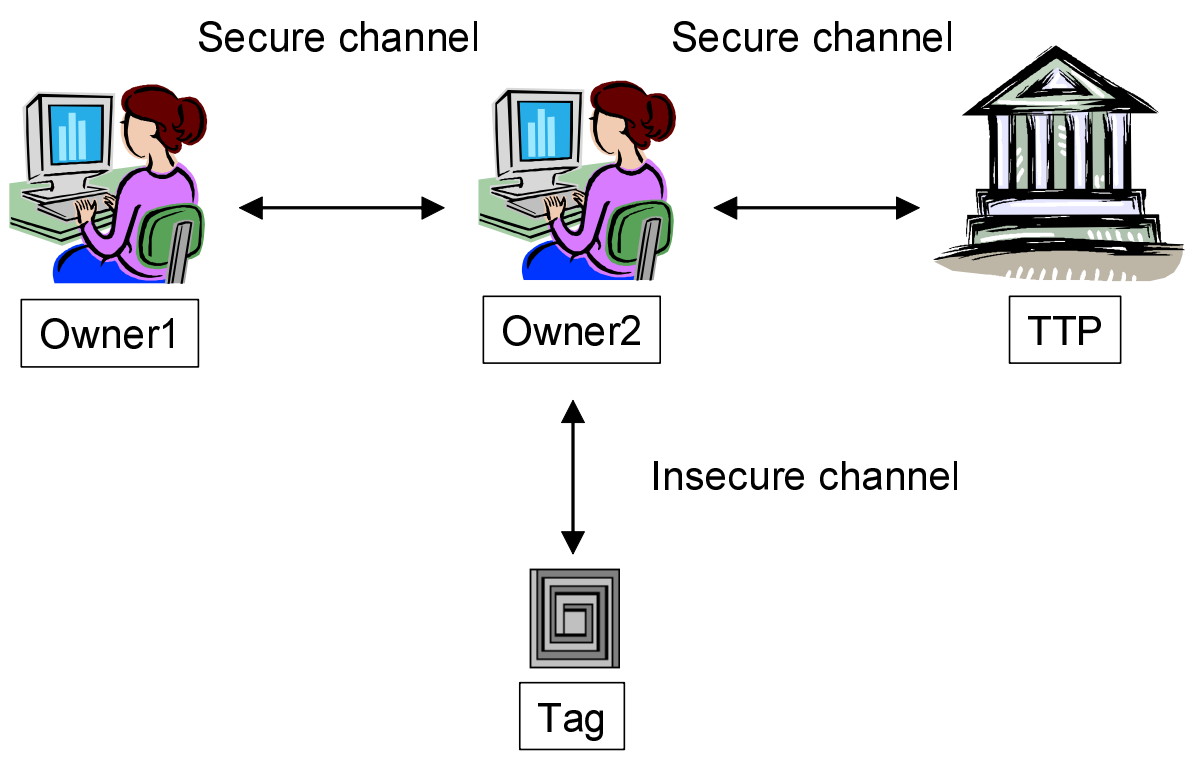

Fig. 2. Model of communication channels

- TTP : Trusted third party. He shares $K_{T T P}$ with an RFID tag $T$. In practice, the role is played by a service provider of traceability or ID provider.

These shared keys are securely shared in advance.

Next, we show our models of communication channels. This model is shown in Figure 2,

- Owner 1 to Owner $_{2}$ : This channel is an existent secure channel. Owner 2 can authenticate $O$ wner $_{1}$ and send a message without leakage.

- Owner $_{2}$ to TTP : This channel is the same as above channel.

- Owner 2 to $T$ : This channel is radio frequency channel. An attacker can tap this channel. Therefore, the channel is not secure.

\subsection{Protocol}

We show our proposed scheme below.

1. Owner $_{1}$ gives a key $K_{1}$ to Owner $_{2}$ by using a secure communication channel.

2. Owner $_{2}$ generates a new key $K_{2}$ and send $K_{1}$ and $K_{2}$ to TTP by using a secure communication channel.

3. TTP generates a ciphertext $C_{T T P}=S K_{K_{T T P}}\left[K_{1}, K_{2}\right]$ by using a key $K_{T T P}$ and send the ciphertext to $\mathrm{Owner}_{2}$.

4. Owner $_{2}$ send the ciphertext $C_{T T P}$ to $T$.

5. $T$ decrypts $C_{T T P}$ by using $K_{T T P}$. If $K_{1}$ is true, $T$ changes the previous key $K_{1}$ to the new key $K_{2}$.

The protocol is shown in Figure 3 . 

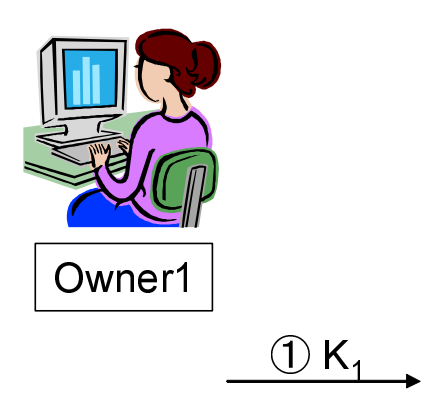
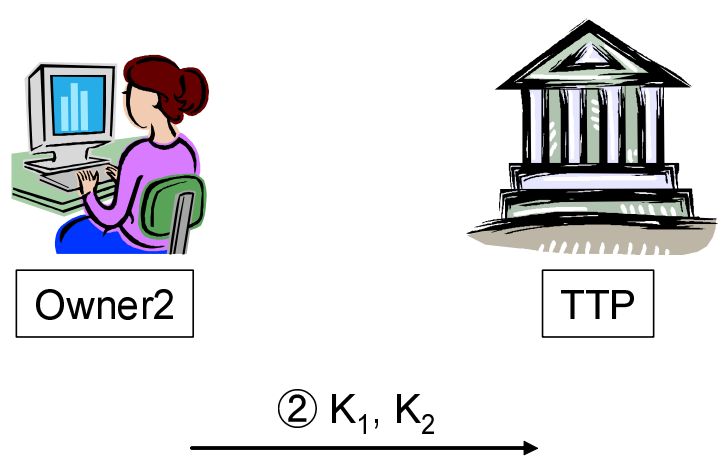

(3) $\mathrm{C}_{\mathrm{TTP}}=\mathrm{SK}\left[\mathrm{K}_{1}, \mathrm{~K}_{2}\right]$

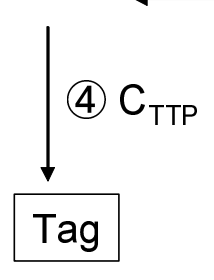

Fig. 3. Owner change scheme in three party model

\subsection{Discussion}

In our scheme, we can change the key on the RFID tag by using TTP. We suppose that the communication channels between previous owner and new owner, and between new owner and TTP are secure respectively. Therefore, an attacker can tap only the communication between new owner and the RFID tag. Moreover, in the communication between new owner and the RFID tag, the new key is encrypted by using the TTP's key which is shared the RFID tag with TTP. Thus, previous owner cannot know the new key because the new key is encrypted by the TTP's key.

However, new owner can know a movement history of previous owner because previous owner must give the own key to new owner in our scheme. To solve this problem, previous owner can change a key which should be sent to new owner. In our scheme, we can change a key by ourselves. Therefore, new owner cannot know the movement history of previous owner because previous owner can change a key to change an owner.

Moreover, we can prevent ID information from leaking because ID information is encrypted by symmetric key encryption. About tracing ID information, we can prevent by changing the key frequently. Since the ciphertext of ID information is changed by changing the key, we can protect location privacy. However, our scheme is not suitable to changing the key frequently because we must communicate with TTP. But, we can protect location privacy by combining our scheme with other privacy protection scheme like [3, 4, 5]. 
Another problem is tampering with the RFID tag. Since the RFID tag has the symmetric key, an attacker can get the key by tampering with the RFID tag. To protect from tampering, we can use tamper-resistant device to keep the key. However, tamper-resistant device is too expensive for the RFID tag to be used for goods management.

\section{Owner Change Scheme on Two Party Model}

Next, we propose an owner change scheme on two party model. This scheme can be also used with other privacy protection scheme like [3,4,5]. In this scheme, we can change a key of an RFID tag without TTP.

\subsection{Model}

We show our model below.

- $T$ : An RFID tag. It has ID information and a key $K_{1}$ which is shared with Owner $_{1}$. It generates a ciphertext $C=S K_{K_{1}}[I D]$ using $K_{1}$. Moreover, it generates a nonce $N$ by using a random number.

- Owner 1 : Previous owner. It shares a key $K_{1}$ with $T$.

- Owner 2 : New owner. It receives $K_{1}$ from Owner ${ }_{1}$ and generates a new key $K_{2}$.

These shared keys are securely shared in advance.

Moreover, we suppose a forward channel and a backward channel [6]. The forward channel is a communication channel from a reader to an RFID tag. It

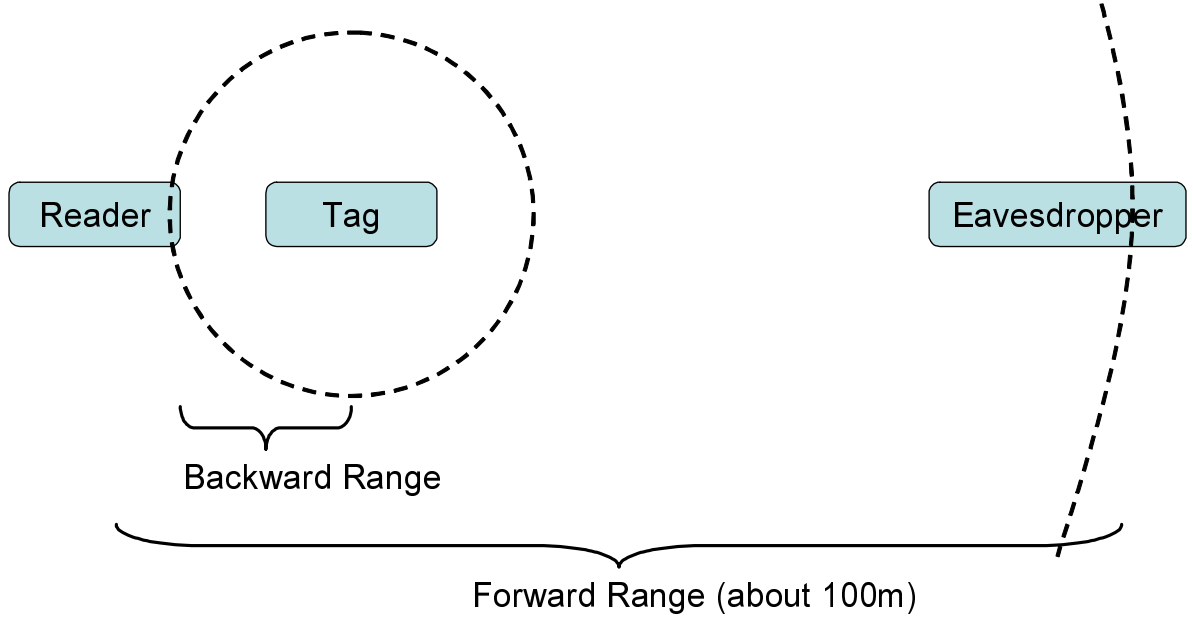

Fig. 4. Forward channel and backward channel 
is relatively long range. On the other hand, the backward channel is a communication channel from an RFID tag to a reader. It depends on the capability of the RFID tag. However, the backward channel is shorter than the forward channel. Therefore, it is more difficult to tap the backward channel than the forward channel. We show the relationship between the forward channel and the backward channel in Figure 4 .

Next, we show our models of communication channels.

- Owner 1 to Owner $_{2}$ : This channel is an existent secure channel. Owner 2 can authenticate Owner $_{1}$ and send a message without leakage.

- Owner $_{2}$ to $T$ : This channel is a forward channel by using radio frequency. It is possible to be tapped by an attacker. So, this channel is not secure.

- $T$ to Owner $_{2}$ : This channel is a backward channel by using radio frequency. It is possible to be tapped by an attacker. However, the attacker must approach to tap this channel because this channel is backward channel. Therefore, this channel is more secure than the communication channel from $\mathrm{Owner}_{2}$ to $\mathrm{T}$.

\subsection{Protocol}

We show our proposed scheme below.

1. Owner ${ }_{1}$ gives a key $K_{1}$ to Owner $_{2}$ by using a secure communication channel.

2. Owner $_{2}$ sends a query to $T$.

3. $\mathrm{T}$ generates a nonce $\mathrm{N}$ and sends it to $\mathrm{Owner}_{2}$.
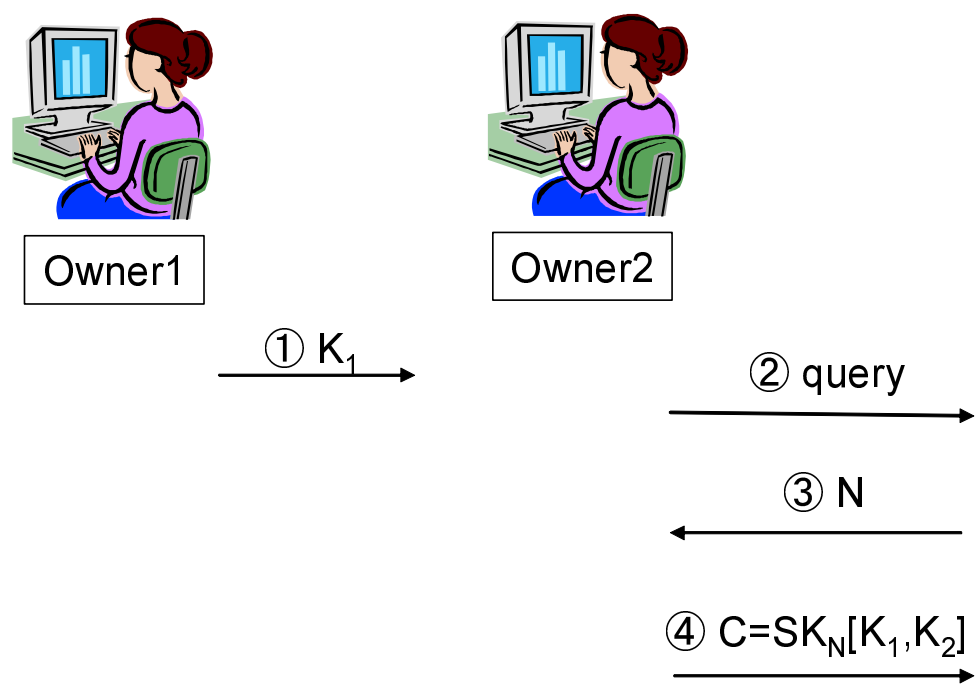

Fig. 5. Owner change scheme in two party model 
4. Owner $_{2}$ generates a new key $K_{2}$ and generates a ciphertext $C=S K_{N}\left[K_{1}\right.$, $K_{2}$ ]. Owner $_{2}$ send the ciphertext $C$ to $T$.

5. $T$ decrypts $C$ by using $N$. If $K_{1}$ is true, $T$ changes a key $K_{1}$ to $K_{2}$.

The protocol is shown in Figure 5 .

\subsection{Discussion}

In our scheme, new owner receives a symmetric key from previous owner and changes the key on an RFID tag by using the key. Moreover, we can protect the new key by using a nonce as an encryption key. When the RFID tag sends the nonce, it is difficult to tap the nonce because the communication is backward channel. However, since backward channel is radio frequency, an attacker can approach and tap the nonce. Therefore, when the RFID tag sends the nonce, we can use more short range communication channel like contact communication.

Moreover, since we can change a key by ourselves, previous owner can change a key to send it to new owner.

\section{Application}

We show application for our schemes. Our schemes are fit to goods management. When RFID tags are used for goods management, its owner changes from a manufacturer to a distributor, and from a retailer to a consumer. A database keeps history of goods movements. So when consumer buy goods, she can get information about a source of goods and operations in manufacture by accessing the database. But she want that the retailer cannot know RFID tags' movement any more. By using our schemes, she can change keys of RFID tags and prevent the retailer from reading RFID tags.

\section{Conclusion}

In this paper, we proposed owner change schemes to protect a new owner's privacy. We proposed two schemes, first is an owner change scheme on three party model and second is an owner change scheme on two party model. In the scheme using TTP, we can prevent a new key from leaking by using a key which is shared an RFID tag with TTP. In the second scheme, we can prevent a key from leaking by supposing backward channel.

As a future work, we will evaluate the cost of implementation of our proposed schemes.

\section{References}

1. A. Juels and R. Pappu, "Squealing Euros: Privacy Protection in RFID-Enabled Banknotes," In R. Wright, editor, Financial Cryptography '03, Spring-Verlag, 2003.

2. Gildas Avoine, "Privacy Issues in RFID Banknote Protection Schemes," International Conference on Smart Card Research and Advanced Applications - CARDIS, Toulouse, 2004. 
3. Junichiro Saito, Jae-Cheol Ryou and Kouichi Sakurai, "Enhancing privacy of Universal Re-encryption scheme for RFID tags," EUC2004, LNCS Vol.3207.

4. A. Juels, "Minimalist Cryptography for RFID tags," Fourth Conference on Security in Communication Networks(SCN'04), 2004.

5. Miyako Ohkubo, Koutarou Suzuki, Shingo Kinoshita, "Cryptographic Approach to a Privacy Friendly Tag," RFID Privacy Workshop, 2003.

6. S. A. Weis, S. Sarma, R. Rivest, and D. Engels, "Security and privacy aspects of low-cost radio frequency identification systems," In First International Conference on Security in Pervasive Computing, 2003.

7. A. Juels, R. L. Rivest and M. Szydlo, "The Blocker Tag: Selective Blocking of RFID tags for Consumer Privacy," ACM Press, 2003. 